\title{
In vitro Synergism of Six Antituberculosis Agents Against Drug-Resistant Mycobacterium tuberculosis Isolated from Retreatment Tuberculosis Patients
}

\author{
Ruoyan Ying ${ }^{1,2}$ \\ Xiaochen Huang' \\ Yaxian Gao ${ }^{1,3}$ \\ Jie Wang' \\ Yidian Liu ${ }^{2}$ \\ Wei Sha ${ }^{2}$ \\ Hua Yang'
}

'Shanghai Key Laboratory of Tuberculosis, Shanghai Pulmonary Hospital, Tongji University School of Medicine, Shanghai, 200433, People's Republic of China; ${ }^{2}$ Tuberculosis Center for Diagnosis and Treatment, Shanghai Pulmonary Hospital, Tongji University School of Medicine, Shanghai, 200043, People's Republic of China; ${ }^{3}$ School of Public Health, Guizhou Medical University, Guiyang, Guizhou Province, 550004, People's Republic of China
Correspondence: Hua Yang Shanghai Key Laboratory of Tuberculosis, Shanghai Pulmonary Hospital, Tongji University School of Medicine, Shanghai, 200433, People's Republic of China Tel/Fax +86 2I 65II5006 ex 3037 Email yanghua97065@tongji.edu.cn

Wei Sha

Tuberculosis Center for Diagnosis and Treatment, Shanghai Pulmonary Hospital, Tongji University School of Medicine, Shanghai, 200043, People's Republic of China

Tel/Fax +86 2l I367|758200

Email shfksw@I26.com
Background: Retreatment tuberculosis (TB) has become a major source of drug-resistant TB. In contrast to the combination of isoniazid (INH) and rifampicin (RIF), that of pasiniazid $(\mathrm{Pa})$ and rifabutin (RFB) or rifapentine (RFP) appears to have better activity in vitro against drug-resistant Mycobacterium tuberculosis (MTB), especially when combined with moxifloxacin (MXF). However, there has been limited study of potential synergism among $\mathrm{Pa}$, RFB, RFP, and MXF, or simultaneous comparison with the standard INH and RIF combination.

Methods: In vitro synergism of four two-drug combinations (INH and RIF, Pa and RFB, Pa and RFP, MXF and Pa) and two three-drug combinations (MXF and Pa combined with RFB or RFP) was evaluated against 90 drug-resistant MTB strains isolated from retreatment TB patients by the checkerboard method. The fractional inhibitory concentration index (FICI) was calculated for each combination.

Results: The synergistic activity of the combination of Pa with RFB or RFP was higher than that of INH and RIF or MXF and $\mathrm{Pa}$, and the synergistic activity of $\mathrm{Pa}$ in combination with RFP was even higher than that of RFB, although RFP yielded an $\mathrm{MIC}_{90}$ of $64 \mathrm{mg} / \mathrm{liter}$, higher than that of RFB of $8 \mathrm{mg} /$ liter against 90 drug-resistant MTB strains. Meanwhile, for three-drug combinations, the synergistic effects of MXF and Pa combined with RFB or RFP were similar. Further stratification analysis showed that, for XDR-MTB strains, the synergistic effect of the Pa and RFP combination was also better than those of other two-drug combinations.

Conclusion: The combination of Pa with RFP shows better in vitro synergism than Pa with RFB and standard INH with RIF combinations, which can provide a reference for new regimens for retreatment $\mathrm{TB}$ patients.

Keywords: Retreatment tuberculosis, XDR-MTB, MDR-MTB, Combined drug sensitivity, FICI, MIC

\section{Introduction}

Tuberculosis (TB) is a chronic infectious disease caused by Mycobacterium tuberculosis (MTB), which requires a long course of treatment and can easily develop drug resistance. ${ }^{1}$ Because of its serious harm to people's health, TB is still a major public health problem throughout the world. Retreatment TB has become a major source of drug-resistant TB, which can evolve into multidrug-resistant TB (MDR$\mathrm{TB})$ or even extensively drug-resistant TB (XDR-TB) if not effectively treated. ${ }^{2,3}$ The current standard chemotherapy regimen for retreatment $\mathrm{TB}$ in China is the recombination and superposition of first-line anti-TB drugs, which is no longer 
suitable to deal with the high drug-resistance rates. ${ }^{4}$ Issues that arise from this regimen are low cure rate, aggravation of adverse reactions, long treatment course, and interruption of treatment with classic regimen, which contribute to the occurrence of MDR-TB. ${ }^{5}$ There is thus a need for new and more effective treatment combinations. In vitro drug susceptibility tests can accurately evaluate the inhibitory activity of different drug combinations against various drug-resistant MTB strains, ${ }^{6-8}$ thereby providing an effective basis for optimizing clinical treatment regimens.

As research on retreatment TB accelerates and more effective treatments are being sought, our previous work introduced a short-range treatment option ${ }^{9}$ by replacing isoniazid (INH), rifampicin (RIF), and streptomycin (SM) in the standard treatment regimen of INH, RIF, $\mathrm{SM}$, ethambutol (EMB), and pyrazinamide (PZA) with pasiniazid (Pa), rifabutin (RFB), and moxifloxacin (MXF) to establish a new regimen of $\mathrm{Pa}, \mathrm{RFB}, \mathrm{MXF}$, $\mathrm{EMB}$, and PZA, which has been applied clinically to retreatment TB patients. ${ }^{9}$ Meanwhile, for patients unable to tolerate the side effects of RFB, rifapentine (RFP) can be used as an alternative. ${ }^{10}$ In vitro activity and synergy between different antimicrobial agents against MTB have been reported. ${ }^{11-13}$ However, the synergism among $\mathrm{Pa}$, RFB, RFP, and MXF against drug-resistant MTB has not been systematically analyzed. In this work, we calculated the antibacterial concentration index or fractional inhibitory concentration index (FICI) of the six core drugs in the two regimens on 90 drug-resistant MTB strains isolated from retreatment TB patients in order to compare the antibacterial effects of the drug combinations of the standard and new regimens. Our results provide further support for the assertion that the combination of $\mathrm{Pa}$ with RFP exhibits better in vitro synergism than Pa with RFB, or INH with RIF, providing a reference for the development of new regimens for retreatment TB patients.

\section{Materials and Methods}

\section{Ethical Approval of the Study Protocol}

The study protocol was approved by the Ethics Committees of Shanghai Pulmonary Hospital of Tongji University (K19-008) in Shanghai, China. It was carried out in line with the ethical standards laid down in the 1964 Declaration of Helsinki and its later amendments. Patients gave their written informed consent to have their data included in this study.

\section{Mycobacterium tuberculosis Isolates}

This study included 90 drug-resistant clinical isolates obtained from retreatment TB patients, including 54 XDRMTB, 29 MDR-MTB, 3 poly-resistant, and 4 INHresistant isolates, at Shanghai Pulmonary Hospital affiliated to Tongji University in Shanghai, China, between January 2011 and December 2015. Drug susceptibility results of the 90 isolates were obtained by the proportion method using the BACTEC MGIT 960 system, as shown in Table S1. The H37Rv (number: ATCC27294) reference strain was also tested. These isolates were stored in $7 \mathrm{H} 9$ broth (Becton Dickinson, Franklin Lakes, NJ) containing $15 \%$ glycerol in a freezer at $-80^{\circ} \mathrm{C}$ until analysis. The test isolates were grown at $37^{\circ} \mathrm{C}$ in Middlebrook $7 \mathrm{H} 9$ broth (Becton Dickson) supplemented with 10\% ADC [5\% bovine serum albumin (BSA), 2\% dextrose, 5\% catalase], and $0.05 \%$ Tween-80 (Sigma-Aldrich) to mid-log phase $\left(\mathrm{OD}_{590} \approx 0.4, \sim 2.5 \times 10^{8} \mathrm{CFU} / \mathrm{mL}\right)$ and diluted with broth medium to a final concentration of $\sim 10^{5} \mathrm{CFU} / \mathrm{mL}$ used for the drug susceptibility testing.

\section{Antimicrobial Agents}

INH, RIF, Pa, RFB, and RFP were purchased from Sigma Chemical Co. (St. Louis, MO); MXF was purchased from Med Chem Express (MCE). Drug configuration: All drugs were weighed to $10 \mathrm{mg}$; Pa dry powder was prepared with $1 \mathrm{~mL}$ of $4 \% \mathrm{NaOH}$ to $10 \mathrm{mg} / \mathrm{mL}$; INH dry powder was prepared with $1 \mathrm{~mL}$ of ultra-pure water to $10 \mathrm{mg} / \mathrm{mL}$; RIF, RFB, and RFP dry powders were dissolved with $1 \mathrm{~mL}$ of dimethyl formamide to $10 \mathrm{mg} / \mathrm{mL}$; and MXF dry powder was dissolved with $1 \mathrm{~mL}$ of dimethyl sulfoxide (DMSO) and prepared to $10 \mathrm{mg} / \mathrm{mL}$. All drug solutions were filtered and sterilized with a $0.22 \mu \mathrm{m}$ sterile filter, and stored separately in a freezer at $-80^{\circ} \mathrm{C}$. Further dilutions were made with coating buffer contained $30 \%$ ethanol and $0.5 \%$ sucrose.

\section{Drug Susceptibility Testing}

Broth microdilution minimum inhibitory concentration (MIC) testing of six anti-TB agents, namely, INH, RIF, Pa, MXF, RFB, and RFP, was performed as described previously. ${ }^{7,8}$ Serial double dilutions of the tested antimicrobial agents were prepared for each isolate using the following ranges of drug concentration: $0.125-8 \mathrm{mg} / \mathrm{liter}$ for $\mathrm{INH}$, 0.03-32 mg/liter for RIF, 0.03-2 mg/liter for MXF, 0.0075$8 \mathrm{mg} / \mathrm{liter}$ for $\mathrm{Pa}, 0.03-2 \mathrm{mg} / \mathrm{liter}$ for RFB, and $0.25-16 \mathrm{mg} /$ liter for RFP, as suggested by the Clinical and Laboratory Standards Institute (CLSI) ${ }^{8}$ with some adjustments. In brief, 
if the standard highest concentration of each drug could not inhibit the growth of the tested isolates, the highest concentration would be doubled; if the tested isolates could not grow under the standard lowest concentration of each drug, the lowest concentration would be halved. The MIC test was performed in duplicate to ensure the reliability of the results.

The checkerboard titration method was used to test the combinations of INH with RIF, Pa with RFB, Pa with RFP, MXF with $\mathrm{Pa}$, and MXF and $\mathrm{Pa}$ combined with RFB or RFP. $^{14,15}$ For the combination of two agents, such as INH (drug A) with RIF (drug B), RIF was serially diluted along the abscissa, while INH was diluted along the ordinate. A1, H12 wells to a 96-well plate (rows A-H, columns 1-12) were prepared as a growth control with drug-free medium. The combination of Pa with RFB, Pa with RFP, and MXF with Pa were prepared as INH with RIF. Three-drug combination checkerboard titration methods were principally based on the standard two-drug (MXF and Pa) combination checkerboard assay. Two-drug combination checkerboard plates were prepared by dispensing the serially diluted $\mathrm{Pa}$ along the abscissa and MXF along the ordinate in a 96-well plate. The third drug (RFB or RFP) was then dispensed on the whole plate with one of the serial concentrations from the MIC range, except the A1 growth control well, as shown in Table 1. For the preparation of drug plates, sterile 96-well plates were added with $20 \mu \mathrm{L}$ of drug-containing coating buffer using an automatic dispenser, air-dried, sealed, and stored at $4^{\circ} \mathrm{C}$. For DST, $200 \mu \mathrm{L}$ of mycobacterial suspension was added to each plate. After incubation at $37^{\circ} \mathrm{C}$ for 2 weeks, the growth status of the drug control strain (H37Rv) and growth control well (wells A1 and H12 in the dual-drug and well A1 in the triple-drug plates) of each plate was observed first to confirm that the drugs were effective and each strain grew normally, after which the MICs of all of the agents in the different combinations were observed and recorded.

The fractional inhibitory concentration index (FICI) of two drugs was calculated by the formula FICI $=(\mathrm{MIC} A+$ $\mathrm{B} / \mathrm{MIC} \mathrm{A})+(\mathrm{MIC} \mathrm{B}+\mathrm{A} / \mathrm{MIC} \mathrm{B})$, where MIC A + $\mathrm{B}$ represents the MIC of compound $\mathrm{A}$ when combined with $\mathrm{B}$; MIC B + A, the MIC of compound $\mathrm{B}$ when combined with $\mathrm{A}$; and MIC A and MIC B, the MIC of compounds A and B tested alone, respectively. Synergy was defined as an FICI value $\leq 0.5$, an FICI value between 0.5 and 4 was considered indifferent, and an FICI value $>4$ was considered to reflect antagonism.

The FICI of three drugs was calculated by the formula $^{15} \mathrm{FICI}=(\mathrm{MIC} \mathrm{A}+\mathrm{B}+\mathrm{C} / \mathrm{MIC} \mathrm{A})+(\mathrm{MIC} \mathrm{B}+$

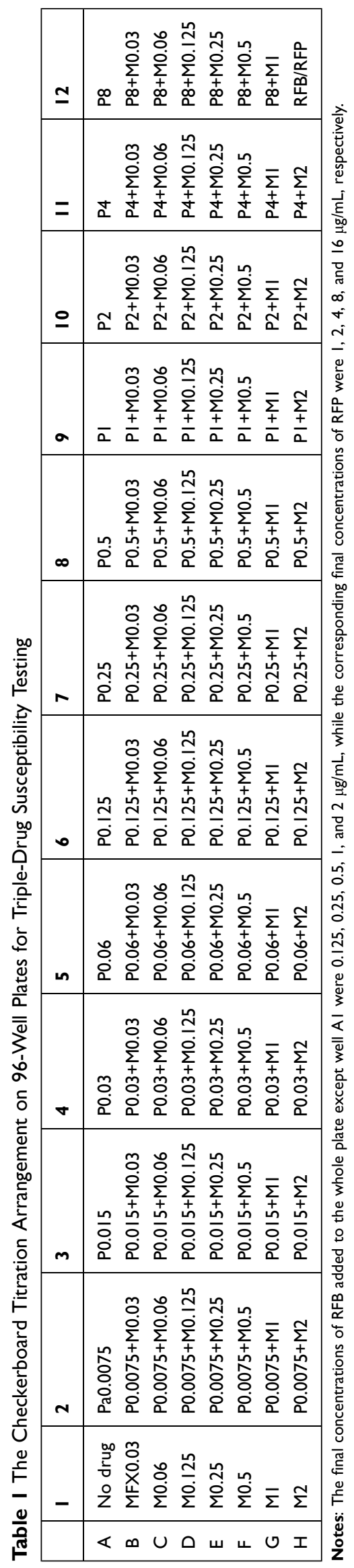


$\mathrm{A}+\mathrm{C} / \mathrm{MIC} \mathrm{B})+(\mathrm{MIC} \mathrm{C}+\mathrm{A}+\mathrm{B} / \mathrm{MIC} \mathrm{C})$, where MIC $\mathrm{A}+\mathrm{B}+\mathrm{C}$ represents the $\mathrm{MIC}$ of compound $\mathrm{A}$ when combined with $\mathrm{B}$ and $\mathrm{C}$; $\mathrm{MIC} \mathrm{B}+\mathrm{A}+\mathrm{C}$, the $\mathrm{MIC}$ of compound $\mathrm{B}$ when combined with $\mathrm{A}$ and $\mathrm{C}$; $\mathrm{MIC} \mathrm{C}+\mathrm{A}+$ $\mathrm{B}$, the $\mathrm{MIC}$ of compound $\mathrm{C}$ when combined with $\mathrm{A}$ and $\mathrm{B}$; and MIC A, MIC B, and MIC C, the MICs of compounds $\mathrm{A}, \mathrm{B}$, and $\mathrm{C}$ tested alone, respectively. An FICI value $\leq 0.75$ was considered to indicate synergism, an FICI value between 0.75 and 4 was considered indifferent, and an FICI value $>4$ was considered to reflect antagonism.

\section{Statistical Analyses}

All experimental data were analyzed using GraphPad Prism software. The $\mathrm{MIC}_{50}$ and $\mathrm{MIC}_{90}$ were used to describe the centralized and discrete trends of the data, and the statistical significance of differences between the groups was determined using the Wilcoxon paired test. Synergy for counting data is expressed as a percentage (\%). Chi-squared test or Fisher's exact test was used to analyze the distribution of FICI values. ${ }^{12}$ A $P$ value $<0.05$ was considered statistically significant.

\section{Results}

\section{Dual-Drug Susceptibility Testing of the} Combinations of INH and RIF, $\mathrm{Pa}$ and RFB, $\mathrm{Pa}$ and RFP, as Well as MXF and Pa

The single- and dual-drug susceptibility test results for the six antimicrobial agents and four combinations against 90 drugresistant MTB clinical isolates from retreatment TB patients are shown in Table 2 and Tables S2 and S3 . Except for the case of MXF, the $\mathrm{MIC}_{50}$ and $\mathrm{MIC}_{90}$ of INH, RIF, Pa, RFB, and RFP were all decreased after the combination of the other drug $(P<0.001)$, which suggests that the combinations of INH and RIF, Pa and RFB, and $\mathrm{Pa}$ and RFP exerted synergistic effects with each other's antimicrobial activity. ${ }^{16}$ After Pa was combined with MXF, the $\mathrm{MIC}_{50}$ and $\mathrm{MIC}_{90}$ of $\mathrm{Pa}$ were reduced from 8 to $0.0075 \mathrm{mg} / \mathrm{liter}$ and 16 to $4 \mathrm{mg} / \mathrm{liter}(P<0.001)$, respectively. However, the $\mathrm{MIC}_{50}$ and $\mathrm{MIC}_{90}$ of $\mathrm{MXF}$ did not decrease after it was combined with $\mathrm{Pa}$, indicating that MXF has a significant unilateral synergistic effect on $\mathrm{Pa}$.

The synergistic, indifferent, or antagonistic effects of each drug combination on the clinical isolates are shown in Table 3 and compared as described in reference 12. The combinations of Pa with RFP and Pa with RFB were more likely to show synergy than that of INH with RIF $(P=0.0127$ and $P<0.0001$, respectively), and MXF in combination with $\mathrm{Pa}(P=0.0004$ and $P<0.0001$,
Table 2 Comparison of MICs for Each Drug Before and After Combinations from Dual-Drug Susceptibility Testing

\begin{tabular}{|c|c|c|c|c|}
\hline \multirow[t]{2}{*}{ Drug Combination } & \multicolumn{3}{|c|}{ MIC (Mg/Liter) } & \multirow[t]{2}{*}{$P$ value } \\
\hline & $50 \%$ & $90 \%$ & Range & \\
\hline \multicolumn{5}{|l|}{ INH+RIF } \\
\hline INH & 4 & 16 & 0.125 to 16 & \\
\hline inh & 2 & 8 & 0.125 to 16 & $<0.001$ \\
\hline RIF & 24 & 64 & 0.03 to 64 & \\
\hline rif & I & 32 & 0.03 to 64 & $<0.001$ \\
\hline \multicolumn{5}{|l|}{ Pa+RFB } \\
\hline $\mathrm{Pa}$ & 2 & 16 & 0.015 to 32 & \\
\hline $\mathrm{pa}$ & 0.5 & 4 & 0.0075 to 16 & $<0.001$ \\
\hline RFB & 4 & 8 & 0.0625 to 8 & \\
\hline $\mathrm{rfb}$ & 0.125 & 2 & 0.03125 to 4 & $<0.001$ \\
\hline \multicolumn{5}{|l|}{ Pa+RFP } \\
\hline $\mathrm{Pa}$ & 2 & 16 & 0.015 to 32 & \\
\hline $\mathrm{pa}$ & 0.25 & 4 & 0.0075 to 8 & $<0.001$ \\
\hline RFP & 32 & 64 & 0.5 to 64 & \\
\hline rfp & 4 & 16 & 0.25 to 16 & $<0.001$ \\
\hline \multicolumn{5}{|l|}{$\mathrm{MXF}+\mathrm{Pa}$} \\
\hline MXF & 0.5 & 1 & 0.03 to 4 & \\
\hline$m x f$ & 0.5 & 2 & 0.03 to 2 & 0.012 \\
\hline $\mathrm{Pa}$ & 8 & 16 & 0.015 to 32 & \\
\hline $\mathrm{pa}$ & 0.0075 & 4 & 0.0075 to 16 & $<0.001$ \\
\hline
\end{tabular}

Notes: INH, MIC of INH tested alone; inh, MIC of INH tested with RIF in combination; RIF, MIC of RIF tested alone; rif, MIC of RIF tested with INH in combination. The rest of the upper- and lower-case letters are the same (dual-drug susceptibility testing); data in bold indicate that the $P$ values were statistically significant.

respectively). $\mathrm{Pa}$ in combination with RFP was more likely to show synergy than the combination of Pa with RFB ( $P=0.0021)$, although RFP yielded an $\mathrm{MIC}_{90}$ of $64 \mathrm{mg}$ / liter, higher than that of RFB of $8 \mathrm{mg} / \mathrm{liter}$.

\section{Triple-Drug Susceptibility Testing (Based on the Combination of MXF and $\mathrm{Pa}$ )}

The dual-drug susceptibility testing provided stronger statistical evidence for synergistic effects for the

Table 3 Distribution and Comparison of $\mathrm{FICl}$ of 90 Strains from Four Dual-Drug Combinations

\begin{tabular}{|c|c|c|c|c|}
\hline FICI Value & INH+RIF ${ }^{a}$ & $\mathrm{~Pa}+\mathrm{RFB}^{\mathrm{b}}$ & $P a+R^{\prime} P^{c}$ & $M X F+P a^{d}$ \\
\hline$\leq 0.5$ & $10(11.1)^{\mathrm{e}}$ & $26(28.9)$ & $45(50.0)$ & $5(5.6)$ \\
\hline $0.5<$ and $\leq 4$ & 80 (88.9) & $62(68.9)$ & $45(50.0)$ & $85(94.4)$ \\
\hline$>4$ & 0 & $2(2.2)$ & 0 & 0 \\
\hline
\end{tabular}

Notes: $P$ value for a vs b comparison was $0.0127 ; P$ value for a vs $c$ comparison was $<0.000$ I; $P$ value for a vs $d$ comparison was 0.2807 ; $P$ value for $b$ vs c comparison was 0.0021 ; $P$ value for $b$ vs $d$ comparison was 0.0004 ; $P$ value for $c$ vs $d$ comparison was $<0.000$ I; e, the data in and outside the brackets represented the percentages and frequencies. 
combinations of $\mathrm{Pa}$ with RFP and $\mathrm{Pa}$ with RFB, while MXF displayed a significant unilateral synergistic effect on $\mathrm{Pa}$. We thus first tested the individual MICs of RFB and RFP in the three-drug combinations (Table S3). The combination of RFB with MXF and Pa decreased the $\mathrm{MIC}_{90}$ of RFB from 8 to $0.2375 \mathrm{mg} / \mathrm{liter}(P<0.001)$, and the combination of RFP with MXF and Pa decreased the $\mathrm{MIC}_{90}$ of RFP from 64 to $1 \mathrm{mg} / \mathrm{liter}(P<0.001)$, as shown in Table 4. The MIC of Pa was also significantly decreased after combination with RFB and MXF, or RFP and MXF $(P<0.001)$. However, the synergistic rates of $\mathrm{Pa}$ and RFB or Pa and RFP after combination with MXF were decreased from $28.9 \%$ and $50 \%$ to $17.8 \%$ and $23.3 \%$, respectively, and the difference between two three-drug combinations was not as significant as for the two-drug combinations $(P=0.5319)$, as shown in Table 5 .

\section{Stratification Analysis}

To evaluate the synergistic effects of different combinations on MTB strains with various drug resistance, further stratification analysis was performed against 54 strains of extensively drug-resistant MTB (XDR-MTB) and 29 strains of multidrug-resistant MTB (MDR-MTB).

Of the 54 XDR-MTB strains tested, the combination of Pa with RFP was more likely to show synergy than those of INH and RIF, MXF and $\mathrm{Pa}$, and $\mathrm{Pa}$ and RFB $(P<0.0001, P<$ 0.0001 , and $P=0.0004$, respectively). The combination of $\mathrm{Pa}$ with RFB was more likely to show synergy than that of MXF and $\mathrm{Pa}(P=0.0001)$, but did not differ from that of INH in combination with RIF ( $P=0.4658)$. The levels of synergism between two three-drug combinations, MXF and Pa combined with RFB or RFP, were similar $(P=0.9646)$, as shown in Tables 6 and 7.

Of the 29 MDR-MTB strains, the combinations of $\mathrm{Pa}$ with RFP and Pa with RFB were also more likely to show synergy than the combination of MXF and $\mathrm{Pa}(P=0.015$ and $P=0.0074$, respectively), but did not differ from INH in combination with RIF ( $P=0.2079$ and $P=0.125$, respectively). The levels of synergism between the two-drug combinations of $\mathrm{Pa}$ with RFP and $\mathrm{Pa}$ with $\mathrm{RFB}$, or the three-drug combinations of MXF and Pa with RFB or RFP, were similar ( $P>0.99$ and $P=0.3847$, respectively), as shown in Tables 8 and 9.

\section{Discussion}

In this study, 90 strains of drug-resistant MTB isolated from sputum specimens of retreatment TB patients were tested for
Table 4 Comparison of MICs for Each Drug Before and After Combinations from Triple-Drug Susceptibility Testing

\begin{tabular}{|c|c|c|c|c|}
\hline \multirow[t]{2}{*}{ Drug Combination } & \multicolumn{3}{|c|}{ MIC (Mg/Liter) } & \multirow[t]{2}{*}{$P$ value } \\
\hline & $50 \%$ & $90 \%$ & Range & \\
\hline \multicolumn{5}{|l|}{$M X F+P a+R F B$} \\
\hline MXF & 0.5 & I & 0.03 to 4 & \\
\hline$m \times f$ & 0.25 & 2 & 0.03 to 2 & 0.129 \\
\hline $\mathrm{Pa}$ & 2 & 16 & 0.015 to 32 & \\
\hline $\mathrm{pa}$ & 0.0075 & 0.5 & 0.0075 to 16 & $<0.001$ \\
\hline RFB & 4 & 8 & 0.0625 to 8 & \\
\hline $\mathrm{rfb}$ & 0.125 & 0.2375 & 0.03125 to $I$ & $<0.001$ \\
\hline \multicolumn{5}{|l|}{$M X F+P a+R F P$} \\
\hline MXF & 0.5 & I & 0.03 to 4 & \\
\hline$m \times f$ & 0.25 & 2 & 0.03 to 2 & 0.719 \\
\hline $\mathrm{Pa}$ & 2 & 16 & 0.015 to 32 & \\
\hline pa & 0.0225 & 4 & 0.0075 to 16 & $<0.001$ \\
\hline RFP & 32 & 64 & 0.5 to 64 & \\
\hline rfp & I & 1 & 0.5 to 4 & $<0.001$ \\
\hline
\end{tabular}

Notes: MXF, MIC of MXF tested alone; mxf, MIC of MXF tested with the combination of $\mathrm{Pa}$ with RFB; $\mathrm{Pa}$, MIC of Pa tested alone; $\mathrm{Pa}, \mathrm{MIC}$ of $\mathrm{Pa}$ tested with the combination of MXF with RFB; RFB, MIC of RFB tested alone; rfb, MIC of RFB tested with the combination of MXF with $\mathrm{Pa}$; The rest of the upper- and lowercase letters are the same (triple-drug susceptibility testing); data in bold indicate that the $P$ values were statistically significant.

Table 5 Distribution and Comparison of $\mathrm{FICl}$ of 90 Strains from Two Triple-Drug Combinations

\begin{tabular}{|l|l|l|}
\hline FICI Value & MXF+Pa+RFB $^{\mathbf{a}}$ & MXF+Pa+RFP $^{\mathbf{b}}$ \\
\hline$\leq 0.75$ & $16(17.8)^{\mathrm{c}}$ & $21(23.3)$ \\
$0.75<$ and $\leq 4$ & $64(71.1)$ & $57(63.3)$ \\
$>4$ & $10(11.1)$ & $12(13.3)$ \\
\hline
\end{tabular}

Notes: $P$ value for a vs b comparison was 0.5319 ; $c$, the data in and outside the brackets represented the percentages and frequencies.

Table 6 Distribution and Comparison of $\mathrm{FICl}$ of 54 XDR-MTB Strains from Four Dual-Drug Combinations

\begin{tabular}{|l|c|c|c|c|}
\hline FICI Value & INH+RIF $^{\mathbf{a}}$ & Pa+RFB $^{\mathbf{b}}$ & Pa+RFP $^{\mathbf{c}}$ & MXF+Pa $^{\mathbf{d}}$ \\
\hline$\leq 0.5$ & $6(1 \mathrm{I} . \mathrm{I})^{\mathrm{e}}$ & $\mathrm{II}(20.4)$ & $28(5 I .9)$ & $3(5.6)$ \\
$0.5<$ and $\leq 4$ & $48(88.9)$ & $4 \mathrm{I}(75.9)$ & $26(48.1)$ & $5 \mathrm{I}(94.4)$ \\
$>4$ & 0 & $2(3.7)$ & 0 & 0 \\
\hline
\end{tabular}

Notes: $P$ value for a vs b comparison was $0.4658 ; P$ value for a vs c comparison was $<0.0001$; $P$ value for a vs $d$ comparison was 0.4862 ; $P$ value for $b$ vs $c$ comparison was $0.0004 ; P$ value for $b$ vs $d$ comparison was 0.000 I; $P$ value for $c$ vs $d$ comparison was $<0.000 \mathrm{I}$; e, the data in and outside the brackets represented the percentages and frequencies.

combined drug susceptibility to accurately evaluate the synergistic effects in vitro of the core drugs from the standard and new regimens. Via the comparison of MIC or FICI from drugs in different combinations, we want to provide some 
Table 7 Distribution and Comparison of $\mathrm{FICl}$ of 54 XDR-MTB Strains from Two Triple-Drug Combinations

\begin{tabular}{|l|c|c|}
\hline FICI Value & MXF+Pa+RFB $^{\mathbf{a}}$ & MXF+Pa+RFP $^{\mathbf{b}}$ \\
\hline$\leq 0.75$ & $8(14.8)^{\mathbf{c}}$ & $9(16.7)$ \\
$0.75<$ and $\leq 4$ & $38(70.4)$ & $37(68.5)$ \\
$>4$ & $8(14.8)$ & $8(14.8)$ \\
\hline
\end{tabular}

Notes: $P$ value for a vs $b$ comparison was $0.9646 ; c$, the data in and outside the brackets represented the percentages and frequencies.

clues for optimizing anti-retreatment TB regimens, especially for drug-resistant TB. ${ }^{17-19}$

INH and RIF are the core drugs in the standard regimen, but many retreatment TB patients have already developed resistance to one or both of these drugs; there is thus an urgent need to develop new regimens for retreatment $\mathrm{TB}$. In previous studies, MXF, Pa, RFB, and RFP were introduced as core candidate options for new regimens for retreatment TB patients. ${ }^{9,10}$ MXF is a fluoroquinolone drug with good bactericidal effects; moreover, it is characterized by mild adverse reactions, high bioavailability, strong tissue permeability, long elimination half-life, and no cross-resistance with other antibacterial drugs, having been broadly used for drugresistant TB. ${ }^{20-22} \mathrm{~Pa}$ is a composite preparation, which is a compound composed of INH and p-aminosalicylic acid; many MTB isolates resistant to INH or p-aminosalicylic acid are still susceptible to $\mathrm{Pa}^{23}$ Both RFB and RFP are

Table 8 Distribution and Comparison of $\mathrm{FICl}$ of 29 MDR-MTB Strains from Four Dual-Drug Combinations

\begin{tabular}{|l|c|c|c|c|}
\hline FICI Value & INH+RIF $^{\mathbf{a}}$ & $\mathbf{P a}+\mathbf{R F B}^{\mathbf{b}}$ & $\mathbf{P a}^{\mathbf{R} F \mathbf{R P}^{\mathbf{c}}}$ & $\mathbf{M X F + P a}^{\mathbf{d}}$ \\
\hline$\leq 0.5$ & $4(13.8)^{\mathrm{e}}$ & $9(3 \mathrm{I} .0)$ & $10(34.5)$ & $\mathrm{I}(3.4)$ \\
$0.5<$ and $\leq 4$ & $25(86.2)$ & $20(69.0)$ & $19(65.5)$ & $28(96.6)$ \\
$>4$ & 0 & 0 & 0 & 0 \\
\hline
\end{tabular}

Notes: $P$ value for a vs b comparison was $0.2079 ; P$ value for a vs c comparison was $0.125 ; P$ value for a vs $d$ comparison was 0.1417 ; $P$ value for $b$ vs $c$ comparison was $>0.99$; $P$ value for $b$ vs $d$ comparison was 0.015 ; $P$ value for $c$ vs $d$ comparison was 0.0074 ; e, the data in and outside the brackets represented the percentages and frequencies.

Table 9 Distribution and Comparison of $\mathrm{FICl}$ of 29 MDR-MTB Strains from Two Triple-Drug Combinations

\begin{tabular}{|l|c|c|}
\hline FICI Value & MXF+Pa+RFB $^{\mathbf{a}}$ & MXF+Pa+RFP $^{\mathbf{b}}$ \\
\hline$\leq 0.75$ & $7(24.1)^{\mathrm{c}}$ & $10(34.5)$ \\
$0.75<$ and $\leq 4$ & $20(69.0)$ & $15(51.7)$ \\
$>4$ & $2(6.9)$ & $4(13.8)$ \\
\hline
\end{tabular}

Notes: $P$ value for a vs b comparison was 0.3847 ; , the data in and outside the brackets represented the percentages and frequencies. derivatives of RIF, ${ }^{24-27}$ and also show cross-resistance to RIF in vitro. ${ }^{28}$ However, studies have shown that RFB and RFP still retain certain bactericidal activity against MTB strains with low resistance to $\mathrm{RIF}^{29}$ and RFB especially has a more significant effect. ${ }^{24,25}$ This is consistent with our results showing that the MIC value of RFB to 90 drug-resistant MTB strains is significantly lower than that of RFP.

Multidrug combination chemotherapy with fluoroquinolones is commonly used for MDR-TB or XDR-TB. In contrast to the study by $\mathrm{Li}$ et al on the synergistic activity of clofazimine with MXF or capreomycin against $30 \mathrm{MTB}$ strains, ${ }^{12}$ we examined the in vitro activity of MFX combined with $\mathrm{Pa}$, RFB and $\mathrm{Pa}$, or RFP and $\mathrm{Pa}$ against 90 drugresistant MTB isolates. After MXF and $\mathrm{Pa}$ were combined, the MIC value of MXF did not change markedly, but the $\mathrm{MIC}_{50}$ and $\mathrm{MIC}_{90}$ of $\mathrm{Pa}$ decreased from 8 to 0.0075 and 16 to $4(P<0.0001)$, respectively, indicating that MXF may be synergistic with the bacteriostatic effect of $\mathrm{Pa}$. However, the synergy rate $(5.6 \%)$ for the combination of MXF with $\mathrm{Pa}$ was similar to that of INH and RIF (11.1\%), as shown in Table 3. This suggests that the combination of MXF with $\mathrm{Pa}$ could not achieve a better antibacterial effect than the INH and RIF combination and that a third drug, especially a rifampin derivative, must be introduced.

Although the detection of drug interaction in MTB by a checkerboard method has previously been reported, ${ }^{6,7,11-13}$ the drug synergism of the two-drug combinations of $\mathrm{Pa}$ and RFB, Pa and RFP, INH and RIF, as well as MXF and $\mathrm{Pa}$, and the three-drug combinations of MXF and $\mathrm{Pa}$ with RFB or RFP against 90 drug-resistant MTB isolates was analyzed for the first time in this work. We found that the combination of $\mathrm{Pa}$ with RFB or RFP had stronger synergistic activity than INH and RIF as well as MXF and Pa. While RFB displayed better antibacterial activity than RFP from the MIC detection results, the combination of $\mathrm{Pa}$ with RFP displayed much better synergistic effects than the combination of $\mathrm{Pa}$ and RFB $(P=0.0021)$. Even for the three-drug combinations, the synergistic effects of MXF and $\mathrm{Pa}$ combined with RFP were also better than those of MXF and $\mathrm{Pa}$ combined with RFB, although the difference was not significant $(P=0.5319)$. Based on our results, we recommend that the combination of MXF and Pa with RFP be used for retreatment TB, instead of the combination of MXF and Pa with RFB. These results further emphasize the importance of analyzing the synergistic effects of drugs in the regimen. 
Stratified analysis further suggested the effectiveness of the combination of Pa with RFP for MTB with different drug resistance profiles. Interestingly, we found no antagonism for the combinations of INH and RIF, MXF and Pa, and $\mathrm{Pa}$ and RFP to all of the strains, while for $\mathrm{Pa}$ in combination with RFB, antagonism was seen only for two XDR-MTB strains. However, the antagonistic rates of three-drug combinations were significantly increased for XDR-MTB and MDR-MTB strains. We hypothesized that the number of drugs and the type of strain in the regimen are important, and that the number of superimposed drugs and different resistant strains might lead to antagonism between drugs in chemotherapy, which also reminds us of the need to consider the combinatorial effects of drugs before formulating a new drug combination regimen.

\section{Conclusion}

This study confirmed that the synergistic effects of the combinations of $\mathrm{Pa}$ with RFB and $\mathrm{Pa}$ with RFP in the new regimen for retreatment TB patients were better than those of INH and RIF or MXF and Pa against 90 drugresistant MTB strains. The bacteriostatic effect of RFB was better than that of RFP, but the synergistic effect of the combination of Pa and RFP was better than that of $\mathrm{Pa}$ and RFB, which can provide a clue in vitro for the design of new regimens for retreatment TB patients. In vitro interactions between core antibacterial agents from the regimen should be detected to ensure the synergistic effects of the drug combinations.

\section{Acknowledgments}

This project was supported by grants from the Chinese National Key Project for Infectious Disease (2018ZX10722302-004 to H.Y.) and the National Natural Science Foundation of China $(81870002,82070007$ to H.Y.).

\section{Disclosure}

The authors report no conflicts of interest in this work.

\section{References}

1. Fekadu G, Chow DY, You JHS. The pharmacotherapeutic management of pulmonary tuberculosis: an update of the state-of-the-art. Expert Opin Pharmacother. 2021;1-10. doi:10.1080/ 14656566.2021.1967930doi:10.1080/14656566.2021.1967930

2. Daley CL, Caminero JA. Management of multidrug-resistant tuberculosis. Semin Respir Crit Care Med. 2018;39(3):310-324. doi:10.1055/s-0038-1661383
3. Seung KJ, Keshavjee S, Rich ML. Multidrug-resistant tuberculosis and extensively drug-resistant tuberculosis. Cold Spring Harb Perspect Med. 2015;5(9):a017863. doi:10.1101/cshperspect.a017863

4. Faridgohar M. Finding new ways to combat multidrug-resistant tuberculosis. Microb Drug Resist. 2020;26(1):71-80. doi:10.1089/ mdr.2018.0353

5. Caminero JA. Management of multidrug-resistant tuberculosis and patients in retreatment. Eur Respir J. 2005;25(5):928-936. doi:10.1183/09031936.05.00103004

6. Yan L, Kan X, Zhu L, et al. Short-course regimen for subsequent treatment of pulmonary tuberculosis: a prospective, randomized, controlled multicenter clinical trial in China. Clin Ther. 2018;40 (3):440-449. doi:10.1016/j.clinthera.2018.01.013

7. Santos NCS, Scodro RBL, Leal DC, et al. Determination of minimum bactericidal concentration, in single or combination drugs, against Mycobacterium tuberculosis. Future Microbiol. 2020;15:107-114. doi: $10.2217 /$ fmb-2019-0050

8. Caleffi-Ferracioli KR, Maltempe FG, Siqueira VL, Cardoso RF. Fast detection of drug interaction in Mycobacterium tuberculosis by a checkerboard resazurin method. Tuberculosis. 2013;93 (6):660-663. doi:10.1016/j.tube.2013.09.001

9. Clinical and Laboratory Standards Institute. Susceptibility Testing of Mycobacteria, Nocardia Spp., And Other Aerobic Actinomycetes. 3rd ed. Wayne, PA: Clinical and Laboratory Standards Institute; 2018.

10. Zhu LZ, Fu Y, Chu NH, et al. [A controlled clinical trial of long course chemotherapy regimens containing rifabutin in the treatment of multi-drug resistant pulmonary tuberculosis]. Zhonghua Jie $\mathrm{He} \mathrm{He}$ Hu Xi Za Zhi. 2006;29(8):520-523. Chinese.

11. Zong Z, Jing W, Shi J, et al. Comparison of in vitro activity and MIC distributions between the novel oxazolidinone delpazolid and linezolid against multidrug-resistant and extensively drug-resistant mycobacterium tuberculosis in China. Antimicrob Agents Chemother. 2018;62(8):e00165-18. doi:10.1128/AAC.00165-18

12. Li G, Xu Z, Jiang Y, et al. Synergistic activities of clofazimine with moxifloxacin or capreomycin against Mycobacterium tuberculosis in China. Int J Antimicrob Agents. 2019;54(5):642-646. doi:10.1016/j. ijantimicag.2019.06.002

13. Coban AY, Bilgin K, Uzun M, Durupinar B. [Effect of linezolid in combination with isoniazid and rifampicin against multidrug resistant Mycobacterium tuberculosis clinical isolates]. Mikrobiyol Bul. 2009;43(2):293-297. Turkish.

14. Rey-Jurado E, Tudó G, de la Bellacasa JP, Espasa M, GonzálezMartín J. In vitro effect of three-drug combinations of antituberculous agents against multidrug-resistant Mycobacterium tuberculosis isolates. Int J Antimicrob Agents. 2013;41(3):278-280. doi:10.1016/ j.ijantimicag.2012.11.011

15. Bhusal Y, Shiohira CM, Yamane N. Determination of in vitro synergy when three antimicrobial agents are combined against Mycobacterium tuberculosis. Int J Antimicrob Agents. 2005;26 (4):292-297. doi:10.1016/j.ijantimicag.2005.05.005

16. Cheng A, Tsai YT, Chang SY, et al. In vitro synergism of rifabutin with clarithromycin, imipenem, and tigecycline against the mycobacterium abscessus complex. Antimicrob Agents Chemother. 2019;63 (4):e02234-18. doi:10.1128/AAC.02234-18

17. Wu X, Yang J, Tan G, et al. Drug resistance characteristics of mycobacterium tuberculosis isolates from patients with tuberculosis to 12 antituberculous drugs in China. Front Cell Infect Microbiol. 2019;9:345. doi:10.3389/fcimb.2019.00345

18. Pontali E, Raviglione MC, Migliori GB. Regimens to treat multidrug-resistant tuberculosis: past, present and future perspectives. Eur Respir Rev. 2019;28(152):190035. doi:10.1183/ 16000617.0035-2019

19. Chae H, Shin SJ. Importance of differential identification of Mycobacterium tuberculosis strains for understanding differences in their prevalence, treatment efficacy, and vaccine development. J Microbiol. 2018;56(5):300-311. 
20. Naidoo A, Chirehwa M, McIlleron H, et al. Effect of rifampicin and efavirenz on moxifloxacin concentrations when co-administered in patients with drug-susceptible TB. J Antimicrob Chemother. 2017;72 (5):1441-1449. doi:10.1093/jac/dkx004

21. Liu Y, Pertinez H, Davies GR, Gillespie SH, Coates AR, Hu Y. Moxifloxacin replacement in contemporary tuberculosis drug regimens is ineffective against persistent mycobacterium tuberculosis in the Cornell mouse model. Antimicrob Agents Chemother. 2018;62(7): e00190-18. doi:10.1128/AAC.00190-18

22. Gillespie SH. The role of moxifloxacin in tuberculosis therapy. Eur Respir Rev. 2016;25(139):19-28. doi:10.1183/16000617.0085-2015

23. Zhang T, Jiang G, Wen S, et al. Para-aminosalicylic acid increases the susceptibility to isoniazid in clinical isolates of Mycobacterium tuberculosis. Infect Drug Resist. 2019;12:825-829. doi:10.2147/ IDR.S200697

24. Johansen MD, Daher W, Roquet-Banères F, et al. Rifabutin is bactericidal against intracellular and extracellular forms of Mycobacterium abscessus. Antimicrob Agents Chemother. 2020;64(11):e00363-20. doi:10.1128/AAC.00363-20
25. Farhat MR, Sixsmith J, Calderon R, Hicks ND, Fortune SM, Murray M. Rifampicin and rifabutin resistance in 1003 Mycobacterium tuberculosis clinical isolates. $J$ Antimicrob Chemother. 2019;74(6):1477-1483. doi:10.1093/jac/dkz048

26. Alfarisi O, Alghamdi WA, Al-Shaer MH, Dooley KE, Peloquin CA. Rifampin vs. rifapentine: what is the preferred rifamycin for tuberculosis? Expert Rev Clin Pharmacol. 2017;10(10):1027-1036. doi:10.1080/17512433.2017.1366311

27. Temple ME, Nahata MC. Rifapentine: its role in the treatment of tuberculosis. Ann Pharmacother. 1999;33(11):1203-1210. doi:10.1 345/aph. 18450

28. Goldstein BP. Resistance to rifampicin: a review. J Antibiot (Tokyo). 2014;67(9):625-630. doi:10.1038/ja.2014.107

29. Rothstein DM. Rifamycins, alone and in combination. Cold Spring Harb Perspect Med. 2016;6(7):a027011. doi:10.1101/cshperspect. a027011
Infection and Drug Resistance

\section{Publish your work in this journal}

Infection and Drug Resistance is an international, peer-reviewed openaccess journal that focuses on the optimal treatment of infection (bacterial, fungal and viral) and the development and institution of preventive strategies to minimize the development and spread of resistance. The journal is specifically concerned with the epidemiology of

\section{Dovepress}

antibiotic resistance and the mechanisms of resistance development and diffusion in both hospitals and the community. The manuscript management system is completely online and includes a very quick and fair peerreview system, which is all easy to use. Visit http://www.dovepress.com/ testimonials.php to read real quotes from published authors. 